\title{
Introduction to TIPS: a theory for creative design
}

\author{
V. V. Sushkov, N. J. I. Mars \& P. M. Wognum \\ Knowledge-Based Systems Group, Department of Computer Science, University of Twente, \\ PO Box 217, 7500 AE Enschede, The Netherlands
}

(Received 15 March 1994; revised version received 22 November 1994; accepted 6 December 1994)

\begin{abstract}
A highly intriguing problem in combining artificial intelligence and engineering design is automation of the creative and innovative phases of the design process. This paper gives a brief introduction to the theory of inventive problem solving (TIPS) selected as a theoretical basis of the authors' research efforts in this field. The research is conducted in the Stevin Project of the Knowledge-Based System Group of the University of Twente (Enschede, The Netherlands) in cooperation with the Invention Machine Laboratory (Minsk, Belarus). This collaboration aims at developing a formal basis for the creation of an automated reasoning system to support creative engineering design.
\end{abstract}

Key words: knowledge-based engineering design, intelligent computer-aided design, creative design.

\section{INTRODUCTION}

Automation of the early phases of engineering design is very important as well as very difficult. The principal objectives of these phases are to design a physically realizable concept of a new engineering system or to improve an existing engineering system in an innovative way. These phases are usually characterized by an illdefined or ambiguous problem statement and the lack of problem solving methods.

The paper presents a method for creative engineering design: the theory of inventive problem solving. An attempt is made to explain why this method was selected as a basis for developing knowledge-based systems to support the creative phases of design. Since the method was developed in the former USSR, it has not been known so far to researchers in the rest of the world who work in the fields of design methodology and computer support for the early phases of design. For this reason, the second objective of the paper is to briefly describe the essential aspects of the method.

\subsection{Cognitive versus systematic approaches to creative design}

In attempts to automate the earliest design phases two approaches are used: cognitive and systematic. Methods based on the cognitive approach, like brainstorming and synectics, suggest psychological ways of enhancing human capabilities in searching for creative solutions. ${ }^{1}$ These methods are very difficult to automate since they do not employ knowledge to constrain the search for solutions and they do not model information about a design product. These methods can be compared with blind search, which results in a large search space and does not guarantee that a satisfactory solution will be obtained. Moreover, evaluation of the solutions resulting from the search is not included.

The methods based on a systematic approach use formal models of design. This implies the construction of a science of design. In a systematic approach, much attention is paid to modeling those aspects of design that are independent of human cognitive abilities. Modeling can be divided into modeling a process of design and modeling a product of this process.

In contrast to the cognitive methods, which also deal with the process of design, systematic ways to model the design process focus on formal methods. An example of a systematic approach to modeling the design process consists of the general principles of design. ${ }^{2}$ These principles explain why one design solution is better than another one and introduce an axiomatic approach to the design process. Although these methods can be successfully used, their principal disadvantage is that modeling an actual design process is largely abstract and subjective and has to be verified in terms of the specific products the process produces.

In the product-oriented approach, the knowledge about the product of a design process is the principal subject of study and modeling. One of the examples illustrating this approach is an ontology for structuring 
and representing product-oriented design knowledge based on system theory and network modeling. ${ }^{3}$ Also, there are several research projects exploring how the bond graphs methodology can be used for modeling design products to assist the designer in the early design phases $^{4}$ or to generate design alternatives. ${ }^{5}$ One of the disadvantages of purely product modeling for conceptual design is the lack of strategic knowledge how to manipulate physical and design information to obtain the best solution.

Successful applications of AI methods based on the use of past experience have also fascinated researchers to apply AI methods for developing computer systems supporting innovative design. ${ }^{6}$ For instance, a popular AI method as case-based reasoning, is used to store, retrieve and use previous design knowledge. ${ }^{7}$ However, the principal weakness of the computer implementations for innovative design based on these methods is that they are strongly limited to a single domain, which makes them inapplicable for problem solving in different domains unless these methods are extended with ways to generalize over domains.

It is argued that the best way to successfully develop a knowledge-based system supporting innovative design is merging the product and process-oriented approaches. As an example, one can envision a computer system for conceptual design ${ }^{8}$ using a combination of the general principles of design and the German design methodologies. ${ }^{9}$

\subsection{TIPS as a theory for creative design}

The objective of this paper is to give an introduction to an approach to creative design which is still not well known worldwide, but seems to be very attractive for combining product-oriented and process-oriented approaches and for developing knowledge-based systems. This approach is based on the theory of inventive problem solving (TIPS). Originally the theory was proposed by the Russian scientist Altshuller ${ }^{10}$ in the early 1950s; and it has been largely extended by his numerous followers since.

The principal advantage of TIPS over other methods for the early design phases is in the depth of the method: TIPS contains high-level knowledge about regularities of engineering system development which have been revealed during many years of analysing world patent collections in various engineering domains. As a consequence, application of the theory is not limited to a single engineering domain. Now, TIPS is a large body of knowledge including various problem solving techniques based on the regularities. It also contains techniques for analysing ill-defined initial situations and extracting a key problem to be solved. ${ }^{11}$

If one compares TIPS to methods for modeling product-oriented design knowledge, the latter are used to model and represent product-oriented design knowledge in a systematic way, but little attention is paid to procedural knowledge on how to manipulate the product models. TIPS contains such procedural knowledge, but little attention is paid to product modeling. As a consequence, from a knowledge-based point of view, both approaches - TIPS and systematic methods for modeling design process - seem to be complementary rather than contradictory. Combining both approaches will make it possible to develop a framework for modeling and representing both types of knowledge.

Most of the literature describing TIPS has been published in Russian. For this reason, the objective of this paper is to show the current state-of-the-art of TIPS. First, it will describe the TIPS philosophy, TIPS structure and explain what is meant by the laws of engineering system development. Then it will concentrate on the techniques introduced by TIPS to solve inventive problems. It will illustrate their practical applicability on several examples in different engineering domains. Finally, it will focus on how knowledgebased techniques can be used for modeling TIPS knowledge.

\section{TIPS PHILOSOPHY}

In the 1950s, Alshuller supposed that the creation of an exact science of the origin and evolution of engineering systems could be done by a comprehensive study and analysis of a large body of information stored in patent collections.

The Soviet system of organizing engineering science simplified this task: in the former USSR, every engineer had almost unrestricted access to the patent collections. This made it possible to involve many researchers and engineers in the investigation. In developing TIPS, Altshuller solicited the collaboration of the invisible college', an informal collection of academic and industrial colleagues throughout the former USSR. The effort was massive, involving hundreds of man years throughout the academic institutions. ' $\ldots$ in the 1980s, about 100 of these institutes and schools are at work. Each year the theoretical principles of the solution of inventive problems are studied by scientists, engineers and students; the volume of "production" is increasing rapidly since the graduates continue to invent after their studies are over. ${ }^{11}$

More than 40 years of studying patents, taken from different areas of engineering, resulted in several important discoveries which form the TIPS philosophy:

(1) All engineering systems evolve according to the same regularities, independently of the domain where they belong to. These regularities can be studied and used for efficient problem solving, as well as for forecasting the further evolution of any engineering system. 
(2) Engineering systems, like social systems, evolve through elimination of various kinds of conflicts. The basic set of principles for eliminating the conflicts is universal for all engineering domains. The origin of the principles is based on the regularities of engineering system development.

(3) Any inventive problem can be represented as a conflict between new requirements and parameters of a prototypical engineering system which is no longer capable of meeting these requirements. Finding an inventive solution to the problem means resolving the conflict under the condition that a compromise is inadmissible.

(4) Often, when searching for an inventive solution to a problem formulated as a conflict, there is the need to use physical knowledge unknown to the domain engineer. To organize and direct the search for appropriate physical knowledge, pointers to physical phenomena should be used. In the pointers, the physical effects are classified according to technical functions which the effects may perform.

A strong claim of TIPS compared to other systematic methods for creative design is that once the modification of an engineering system has been done in accordance with the general regularities of engineering systems development, the modification can be regarded as the most progressive improvement of the engineering system. The use of these regularities makes it possible to significantly restrict the space of possible alternative solutions and to guarantee finding the best solution along the line of engineering system evolution.

\section{INVENTIVE PROBLEM AND INVENTIVE SOLUTION}

In TIPS, a distinction is made between inventive and routine ways of solving design problems. An inventive approach to problem solving is required when it is unclear how to solve the problem using the design and physical knowledge available in the domain in which the problem arose. TIPS defines basic features of an inventive problem:

(1) In the inventive problem, a conflict arises between a system parameter which should be improved to meet the requirements and another system parameter which inadmissibly deteriorates as a result of the improvements.

(2) Optimization of the parameters based on using the available domain knowledge does not produce a desired solution. This makes finding a solution to the problem very difficult, since it is unclear in what area of knowledge to organize the search.

The concepts of an inventive problem and an inventive solution will be illustrated by a real example from the 1960 s after Altshuller had completed the first collection of inventive principles for resolving conflicts.

Example. In a glass manufacturing organization, the following problem arose: melted glass had to be transported during the manufacturing process, but the transporting mechanism (rotating rollers) unacceptably distorted the glass surface. Attempts to solve the problem using the knowledge available in the glass industry did not suggest satisfactory results, because all suggested solutions significantly reduced other parameters of the manufacturing process. For instance, the use of an additional mechanism for smoothing the surface after transporting led to a significant complication of the system.

To solve this problem the following requirement was formulated: transporting the melted glass should be done without damage to its surface. The specification of the conflict 'any improvement available in the domain of processing the glass surface significantly complicates the manufacturing process' was needed to establish a reference to an appropriate inventive principle from the TIPS collection of inventive principles. In the case mentioned, the principle of transition to the microlevel was applied, and one of the possible solutions was to use a flow of melted pewter in place of the rollers (the solution originally was found by Altshuller with the help of TIPS).

As seen in the example, to find a solution to the problem was very difficult because the principle of using melted pewter (or using any kind of melted metal for transportation) was unknown in the domain of glass manufacturing. An inventive solution often lies outside the domain where an engineer works. As a consequence, to solve a problem in an inventive way requires organizing transfer of knowledge from one engineering domain to another. In addition, since the search is organized within a large diversity of knowledge, an efficient method to restrict the search should be used.

Therefore, TIPS can be regarded as a method to organize and direct the search for transferable design knowledge in the phase of creative design. In contrast to AI methods for reuse of previous design knowledge based on case-based reasoning where a new solution is obtained by reusing and adapting design cases from a certain engineering domain, TIPS principles are ahstractions of design cases from various domains.

\section{TIPS STRUCTURE}

As shown in Fig. 1, TIPS is a system of knowledge to support creative design. TIPS is structured into several parts according to the aims of each technique and the 


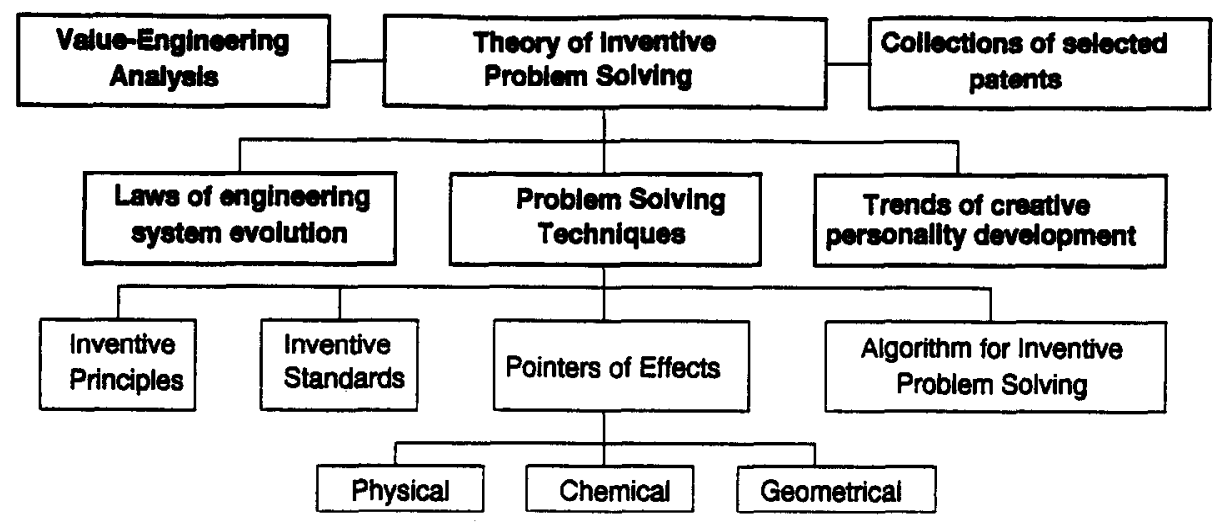

Fig. 1. TIPS structure.

type of problem model with which the technique operates. Among the principal TIPS parts are:

(1) Laws of engineering system evolution. This part of TIPS describes the general regularities of engineering system evolution.

(2) Problem solving techniques. The techniques are aimed at modeling a problem and at producing recommendations as to in what direction to solve the problem.

(3) Collection of selected patents. This part contains patent descriptions selected from various engineering domains. The patents are classified in accordance with the laws of engineering system evolution. They are used to find analogous design cases in different domains.

(4) Value-engineering analysis (VEA). TIPS suggests an extended version of the approach to VEA. ${ }^{12}$ The main extension is that the TIPS version of VEA includes a new algorithm for functional analysis. The output of TIPS-VEA is a set of problems in the form required to be solvable by means of TIPS problem solving techniques. ${ }^{13}$

(5) Trends of creative personality development. This part concentrates on studying psychological methods to enhance creative thinking and teaching creativity to persons as early as possible.

The next section will focus on the laws of engineering system development and section 6 on TIPS problem solving techniques since they will be used in the development of a design support system.

\section{LAWS OF ENGINEERING SYSTEM EVOLUTION}

The theoretical background behind TIPS is formed by the laws of engineering system evolution. The laws were formulated by Altshuller and his research team after analysing hundreds of thousands of patents and many bibliographical sources containing the history of the development of engineering. The laws indicate universal trends in engineering system evolution. TIPS states that any engineering system obeys those laws independently of the domain to which it belongs. As a consequence, the applicability of the laws is not restricted to a single domain, they are correct for the entire engineering domain.

\subsection{The law of system ideality}

The basic TIPS law is the law of system ideality. The law indicates a principal design requirement which every designer has to keep in mind while creating a new engineering system: the system has to perform every desired function whereas the expenditures required to provide the system life-cycle should be as minimal as possible. The law is formulated in the following way: 'system ideality is a ratio between the useful functionality performed by the system and expenditures necessary to fulfill this functionality'. The expenditures in this definition are all kinds of energy, material and informational resources necessary to perform the functionality. However, in every engineering system this ratio tends to increase during evolution of the system.

Example of an ideal solution. When developing a portable radio station for mountain climbers the problem of ensuring temperature stabilization of the oscillator's quartz crystal arose. Instead of using a conventional thermostabilizer which is too heavy and needs a special power source, it was suggested fixing the crystal on the mountain climber's body. Hence, the function of temperature stabilization is fulfilled with no special design at all.

\subsection{Other TIPS laws}

At the moment TIPS counts nine laws of engineering systems evolution. ${ }^{14,15}$ They are divided into three groups: laws of statics, laws of kinematics and laws of dynamics. This division was made to indicate that applicability of the laws depends on the stage of the system evolution. 
Example. One of the laws of dynamics is the law of transition to the microlevel. It states that in the final stage of the system's development, a basic physical effect responsible for performing some system function is replaced with a new effect capable of performing the same function by using field interactions instead of mechanical actions. For instance, instead of mechanical cutting of materials a laser beam can be used.

The laws of statics belong to the initial stage, when the system has just been designed; the laws of kinematics are used during the stage of further development of the system. When features of the laws of dynamics are observed, this indicates that the system has come to a final stage of existence, and will soon be replaced with a new, more effective system, based on a new physical principle of action.

Example. The law of making a system more dynamic states that every design has a trend to increase the number of degrees of freedom within the system.

The following two technical solutions illustrate this law. The first solution is taken from the aircraft industry: a nozzle of a jet engine is made as a telescopic pipe. While operating, the nozzle is pulled out to its fullest extent, and pulled in for transportation (US Pat. 3561679). Another solution is taken from the optical industry: a mirror with changeable geometry. A sectioned pneumatic chamber made of flexible material is placed behind the mirror. The curvature of the mirror surface can be changed by the varying pressure in different sections of the chamber.

The power of the laws is that they can be used to indicate the period of the system evolution as well as to predict how the system should be further developed. Therefore, the laws can be regarded as independent techniques for problem statement and solving. However, with little experience in TIPS their applicability is rather difficult. For this reason, they were smoothly incorporated into TIPS problem solving techniques that makes their use more convenient. An overview of TIPS problem solving techniques with some examples is given in the next section.

\section{TIPS TECHNIQUES FOR PROBLEM SOLVING}

After Altshuller formulated the basic principles of inventive design, they were further elaborated by him and his research team and divided into three TIPS problem solving techniques:

(1) Inventive principles for elimination of engineering conflicts.

(2) Inventive standards, where a standard means that there is some common inventive way of problem solving for certain classes of inventive problems.
(3) Scientific-engineering effects, which are used to organize the reuse of physical knowledge in inventive activities.

TIPS also contains an integrating technique called 'algorithm for inventive problem solving' (AIPS). AIPS includes rules for extracting a root problem from illdefined inventive situations, rules for writing a problem statement in terms of a conflict, and principles for elimination of the conflicts. AIPS also contains rules to control the use of the above mentioned techniques.

Below, each problem solving technique will be discussed separately. AIPS is discussed in section 7 .

\subsection{Principles for elimination of engineering conflicts}

One of the first TIPS techniques developed for solving inventive problems was a collection of so-called principles for elimination of engineering conflicts. An engineering conflict arises when an attempt to improve some technical parameter of a system causes unacceptable deterioration of another system parameter (for instance, attempts to increase the speed of a car lead to the growth of fuel consumption). An inventive principle is a heuristic rule that can be used to produce a recommendation on how to solve a problem without causing unacceptable negative effects.

From his study of the patents collection, Altshuller has found that there are 39 generalized engineering parameters, like the weight of a movable object, which are abstractions of various specific engineering parameters. The problem can be solved by the use of some principle after the problem model has been reformulated as an engineering conflict: a generalized parameter to be improved versus a generalized parameter which deteriorates. The principle itself does not give a solution to the problem, it recommends a direction for eliminating a certain type of engineering conflict.

At the moment, 40 inventive principles aimed at resolving conflicts between generalized parameters are known. The principles are accessible through indices in a matrix. Along the vertical axis of this matrix the generalized parameters that have to be improved are specified; along the horizontal axis the parameters that deteriorate while improving the first are specified. These parameters can be looked up along the vertical and horizontal axes and the matrix suggests up to four principles that can be used to solve the conflict. Selected principles are ordered according to their applicability. The principle that will most likely solve the problem is given first (Fig. 2).

Example. The weight of a short steel pipe is small enough and does not hinder movement of the pipe inside a kiln during thermal processing. However, to process a long pipe is more difficult: its large weight hinders the transportation. In this situation, a conflict arises 


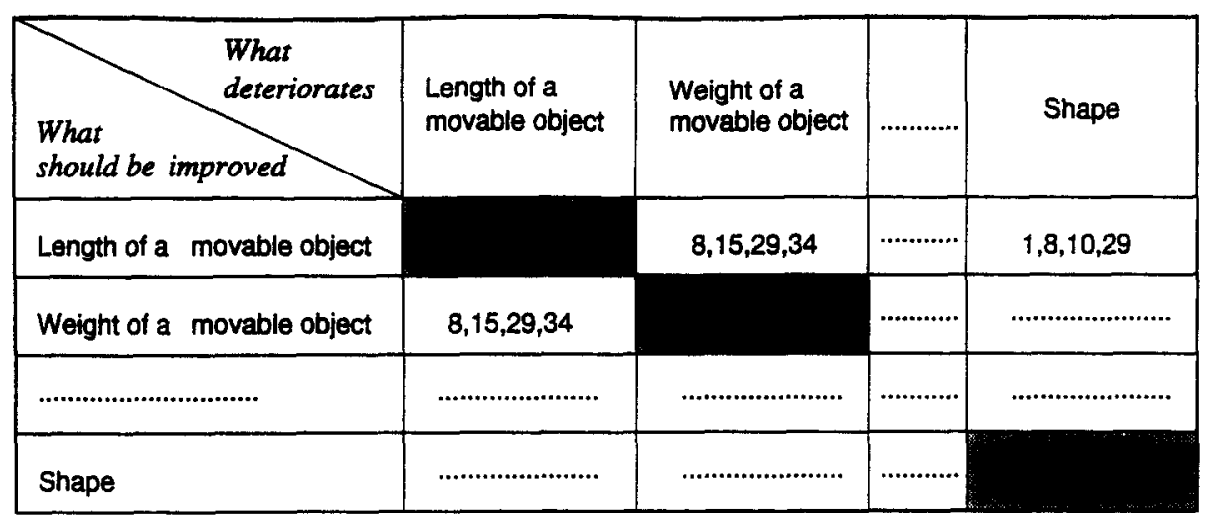

Fig. 2. A table of principles for engineering conflict elimination. Inventive principles: 1, principle of segmentation; 2 , principle of antiweight; 10 , principle of preliminary action; 15 , principle of making a system more dynamic; 29 , use of pneumatic and hydraulic structures; 34, principle of rejection and regeneration of system parts.

between the parameters 'length of the movable object' and 'weight of the movable object'. One of the inventive principles suggests using pneumatic and hydraulic structures to eliminate this kind of conflict. One of the known solutions to the problem is to create an air cushion in the kiln, which provides the required movement of long pipes.

\subsection{Inventive standards}

Another TIPS problem solving technique is a collection of inventive standards. The term inventive standard means that there is some common problem-solving method applicable to the whole group of similar inventive problems. If problems taken from different domains result in identical problem models, this means that the problems are similar, and they can be solved in the same way.

The collection of inventive standards resulted from further development of the principles, and include their best features. In contrast to inventive principles which operate with generalized technical parameters, standards operate with a physical model of a design where a problem arose. This makes the standards more specific than the principles.

To model the physical structure of a design where a problem arose and which is responsible for providing the required functionality, substance-field modeling is used. The basic idea behind the substance-field modeling is that any part of an engineering system can be represented as a set of substance components and field interactions between the components. The problem is indicated as an undesirable, insufficient or missed interaction. To obtain a solution to the problem means that the initial physical structure which contains an unsatisfactory or missing interaction has to be transformed into a structure in which the desired behavior is achieved. The standards specify a way in which such a transformation can be achieved.

There are two basic physical principles to change the physical structure of any engineering system, which are used in the standards:

- Any physical attribute of a substance component can be changed by introducing a field affecting this substance and capable of changing the attribute.

- Any attribute of a field in the required place of a system can be changed by a substance component, introduced into this place and capable of changing the field attribute.

The inventive standard is a rule, in which the left part specifies the conditions of a problem, that is, what restrictions there are on the introduction of additional components and of what kind is the substance-field model of the problem. The right part specifies how the model should be transformed to meet the requirements. The standard itself does not exactly specify the substances and fields to be introduced and how they should be introduced into a system; the standard only indicates an abstract method of transformation.

As follows from the nature of the standards, substance-field modeling is conducted at a very abstract level, without specifying specific values of system parameters. Important are the interacting substance components and a field providing an energy flow from one component to another. The problem is specified in terms of the attributes of the components or the field to be altered.

Example. A system for melting glass ampoules filled with a liquid medicine consists of a nozzle producing a flame which melts the ampoule tips, and a container in which the ampoules are stored. A problem arises because of the difficulty of supporting the necessary length of the flame: when the flame becomes larger than required, it unacceptably overheats the medicine. The substance-field model of the problem is depicted in Fig. 3 (right side). The arrows show the direction of actions provided by the fields.

To solve the problem, the following standard was used: STANDARD 1-2-1. If a useful and a harmful 


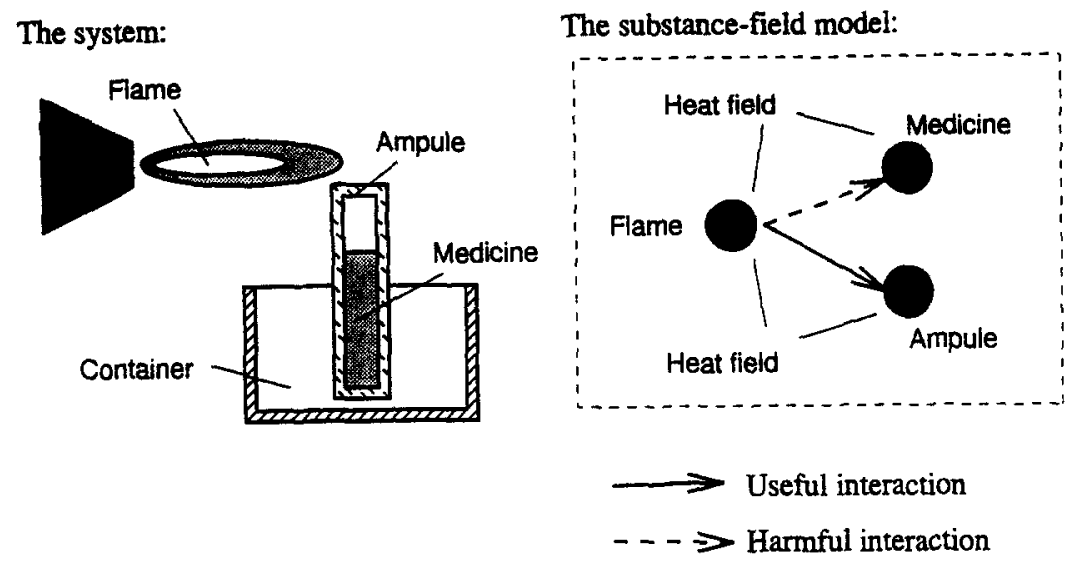

Fig. 3. Substance-field modeling of the problem of melting the ampoules.

effect appear between two substances in a substancefield model, with no need to maintain direct contact between the substances, the problem is to be solved by introducing a new substance, either free or quite cheap, between them.

One of the recommended solutions is to fill the container with an incombustible liquid, e.g. water, protecting the lower part of the ampoule containing the medicine, whereas the upper part is to be kept above the water surface. In this case the need to control the flame ceases.

The complete system includes 76 standards. The standards are subdivided into five classes. Each class includes several standards collected together depending on the type of problem (in particular, an initial substance-field model).

Besides the standards operating with substance-field models, the system of standards contains a set of standards using various aspects of the laws of engineering system evolution which are applicable for problem solving. However, these standards are still very informal, so their use is quite difficult.

Example. Standard 2-3-1. The efficiency of a substance-field system can be enhanced by matching (or mismatching) the frequency of a field's action with the natural frequency of a product (or a tool).

\subsection{Scientific-engineering effects}

The third technique suggested by TIPS consists of the collection of scientific-engineering effects. Studies of the patent collections indicated, that the best and, as a consequence, more ideal inventive solutions were obtained by using natural phenomena. Knowledge of the natural phenomena used to invent a new system or to improve an existing system often makes it possible to avoid the development of a complex design. For instance, instead of using a mechanical design including many parts for the precise displacement of a working tool for a short distance, it is possible to apply the effect of thermal expansion to control the displacement.

Finding a relevant natural phenomenon which is capable of meeting a new engineering requirement should be regarded as one of the most important tasks in the early phases of design. However, it is nearly impossible to use formulations of natural phenomena as given in physics or chemistry for the process of generating various design alternatives. The descriptions of physical phenomena yield information on the properties of interacting substances and fields from a scientific point of view, and it is unclear how these properties can be used to achieve specific technical functions.

The TIPS collection of physical effects is designated to bridge the gap between science and engineering. Thus, the definition of the scientific-engineering effect implies that each physical effect in this collection is associated with a multitude of various technical functions the effect may perform. In turn, applicability of each effect is illustrated by alternative design implementations.

All the natural effects are classified into three large groups according to the field of science to which they belong: physical, chemical and geometrical. The search for an effect is possible through indicating the engineering requirement to be performed by a new design. These requirements are collected into the list of so-called technical functions. Each technical function is a purpose that can be achieved in a system, such as to move a loose body or to change a density. Each of these technical functions refers to a list of several possible effects which can be used, which makes it possible to apply well-known effects in non-ordinary situations. The organization of the collection is shown in Fig. 4.

Example. The function to achieve a precise displacement refers to the physical effect of magnetostriction in the TIPS database of effects. The practical use of this effect for performing the function is illustrated by solving the problem of achieving a precise distance between a magnetic head and a recording surface in a 


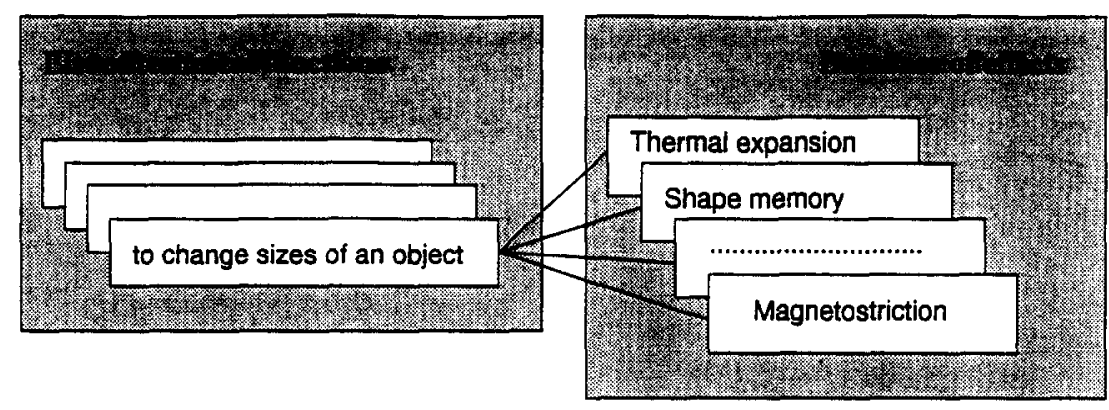

Fig. 4. The organization of the effects collection.

tape recorder. One end of the magnetostrictive rod is fixed in a rigid position and the opposite end is fixed to the head. A magnetic field is applied to compress the rod exactly to the required distance between the head and the recording surface (SU A.c. 517 927).

The method of organizing the TIPS collections of scientific-engineering effects is similar to the approach used by the German School of Design to develop Design Catalogues. ${ }^{9}$ However, there are two distinctions between the TIPS collections of effects and the Design Catalogues: first, TIPS understands a technical function in the broader sense, and any physical function is regarded as generic with respect to producing a multitude of technical functions; second, TIPS contains collections of geometrical and chemical effects which relate chemical and geometrical knowledge to technical functions. ${ }^{16}$ The collections of geometrical and chemical effects are organized in the same way as the system of physical effects.

Example. Many of the technical functions can be performed by using various geometrical shapes. For instance, the function to obtain different properties on one side of an object can be provided with the use of a Möbius sheet. The Möbius sheet is a twisted sheet, glued in a ring. While rotating, it has a one-sided surface, whereas actually it has a two-sided surface. By covering both surfaces of the sheet with different materials, it is possible to obtain two different properties on one side of the sheet.

The relation between the effects, principles and standards is not always clear. Accidentally, standards and principles may correspond to the desired results and thus act as indices for the collections of effects. The effects can best be viewed as a useful collection of natural science knowledge to support the other problem solving methods.

\section{AIPS}

This section discusses the algorithm for inventive problem solving (AIPS): an integrating technique designated to solve complex inventive problems. The first version of AIPS was developed in 1956, and has been largely extended and modified since. ${ }^{17}$ Below, will be discussed the essential parts of the latest AIPS version known as AIPS-85B. ${ }^{14}$

\subsection{Limitations of TIPS problem solving techniques}

Although all three techniques discussed above are rather powerful and may be successfully used, one can distinguish, at least four disadvantages:

(1) The techniques operate with fuzzy information about an engineering system and its surrounding environment. They are not supported by tools for verifying a problem statement. As a consequence, a model of a problem is constructed ad hoc.

(2) An ill-defined inventive situation can be decomposed into various engincering conflicts and substance-field models. There is no tool to select the right model from a number of alternatives.

(3) Sometimes, when a difficult problem is only analysed in terms of a conflict between engineering parameters or substance-field interactions, the solution may not be found.

(4) The TIPS problem-solving techniques do not remove psychological inertia: specific engineering terms shackle an inventor to ingrained concepts about technical objects, thus significantly complicating the search for new solutions.

Attempts to eliminate these disadvantages resulted in the development of the AIPS. AIPS-85B consists of specific techniques aimed at supporting all phases of the problem solving process with TIPS: from the initial problem statement to the qualitative verification of obtained solutions. Besides, AIPS includes specific rules intended to help with applying the TIPS problem solving techniques discussed in the previous section and a large collection of various industrial solutions accumulated during the long-term development and use of TIPS. Many of the solutions provide detailed information on how the solution was obtained. Thus, they can be viewed as previous cases storing inventive experience with tackling difficult situations occurring in different steps of problem solving. This information is used as guidance in solving a new problem. 


\subsection{Physical conflicts}

There might be two undesirable situations after applying TIPS problem solving techniques:

(1) Numerous attempts to solve a problem do not produce the required result.

(2) A solution to the problem found with the help of inventive standards or the collections of effects may not be accepted with respect to the problem constraints or environmental demands. Attempts to find an acceptable solution fail.

These situations indicate that the actual origin of the problem has not been extracted during the step of problem analysis. Inventive principles and inventive standards are supposed to only conduct a superficial analysis of the problem whereas, in many situations, the most difficult inventive problems are featured by a physical conflict: the same component of an engineering system should have different values of some physical parameter or should be in different physical states at the same time.

Example. A window glass should be transparent to a light beam and should not be transparent to heat; a temperature of a flame should be large enough to provide the welding of objects and should be small enough to prevent overheating of the objects being welded.

To reveal the physical conflict, more sophisticated problem analysis is needed. For this reason, AIPS focuses on modeling a problem as a physical conflict and resolving this kind of conflict.

In contrast to procedures for formulating the engineering conflict and substance-field modeling, the physical conflict often may not be directly extracted from the initial problem description. To translate the initial problem statement into the physical conflict, AIPS suggests several steps, each of which includes special rules for constructing and verifying the physical conflict. To help with problem solving after the physical conflict has been successfully extracted, AIPS introduces principles for the elimination of physical conflicts.

In the next subsections will be described the AIPS structure and a case study illustrating problem solving by AIPS.

\subsection{AIPS: a case study}

In this section, it is demonstrated how AIPS helps with solving a real design problem. To make an understanding of the basic AIPS concepts more clear, a problem solving process by AIPS will be divided into three steps:

- analysis of an inventive situation and problem formulation
- problem modeling

- problem solving

\subsubsection{Analysis of an inventive situation and problem formulation}

The first step of AIPS is an analysis of an inventive situation and decomposition of the initial situation into manageable subproblems. A selection of a problem to solve from the subproblems is made according to the following rule: a subproblem that involves minimal modifications to obtain a solution should be selected.

Example. Consider the following inventive situation: to indicate the level of a combustible liquid in a container, a float detector is used. When the level of the liquid reaches the upper part of the container, a contact placed on the float and the metal surface of the container upper part close a circuit and a signal is generated (Fig. 5). However, when there is a small gap between the float contact and the surface of the container, a spark jumps across them, which can cause combustion of the liquid. In this case, there is an inventive situation which can be decomposed into a set of subproblems: to prevent the spark jumping, to isolate the gap from the liquid, to replace an electrical system with some other system, etc., without knowing which problem to solve.

As said above, AIPS recommends solving a miniproblem: a solution should be found with minimal modifications of the existing system. The problem is formulated in the following way. An engineering system for providing float detection includes: the float, the combustible liquid, the container, the contacts, the conductors, a voltage source, electrical current and a lamp. It is necessary to prevent the spark jumping with minimal system modifications.

\subsubsection{Problem modeling}

After the mini-problem has been formulated, it is modeled as an engineering conflict. In AIPS, the formulation of an engineering conflict slightly differs from the formulation used in the inventive principles (Section 6.1). The AIPS formulation of the engineering conflict consists of two parts. Both parts indicate opposite situations of the conflict and the relevant positive and negative effects produced in both situations.

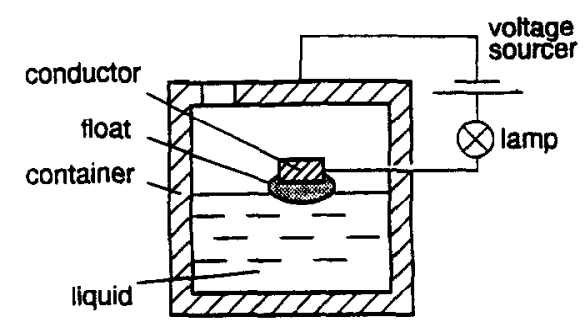

Fig. 5. A container with combustible liquid and a level detector. 
Example. The engineering conflict corresponding to the mini-problem formulated in the previous section is formulated in the following way:

- Part 1. An electrical current can be used to indicate the liquid level, but the liquid can ignite as a result of a spark jumping.

- Part 2. Without a current, the spark does not appear, but level detection is not provided.

After this step has been completed, AIPS suggests constructing a substance-field model of the problem and to attempt to find a solution based on inventive standards. However, even if the solution is found, one needs to continue the work with AIPS because there is no guarantee that the best possible solution has been found.

The next step is an analysis of the available substance-field and other resources. Cheap available resources should be used as much as possible to solve the problem against the lowest possible cost. At this step, the ideal final result is formulated. The ideal final result displays the ultimate goal of problem solving: the desired result should be obtained without introducing additional expensive matter into the engineering system. This also means that all components to be introduced to construct a solution should be either available resources or derived from them.

Finally, after a comprehensive analysis of the problem has been done, the engineering conflict is transformed into a physical conflict. There are two kinds of physical conflicts: a macro-level conflict and a micro-level conflict. The macro-level conflict is modeled as contradicting demands on the physical state of some component in the system. The micro-level conflict specifies exactly where and when the particles of a substance should provide a required useful effect and should not be a cause of a negative effect.

Example. The macro-conflict of the problem is: an electrical current should be in the system to provide detection and must be absent to prevent the spark jumping.

The micro-level conflict is: an electrical current in the system should only exist when the contacts are closed and should not exist when the contacts are opened.

\subsubsection{Problem solving}

The problem solving phase consists of two parts:

(1) Decide on what should be done to modify the existing system by means of using the principles for physical conflict elimination.

(2) Find how this modification can be achieved by using the collections of physical effects to retrieve physical knowledge which will make the solution physically realisable.
Example. One of the principles for the elimination of physical conflicts states that the conflict formulated at the previous step can be resolved by the division of contradictory properties in time. Thus, one obtains a new formulation of the problem: the electrical current has only to be generated when the contacts are closed, and must not be generated when the contacts are opened.

To solve the problem in such a formulation, after using the collection of physical effects it was suggested using the Seebeck effect: appearance of electromotive force in contacting heterogeneous metals with different values of thermopowers. One of the possible solutions (Fig. 6) is based on this effect: the container and the conductor placed on the float are made of different metals. The metal of which the container is made has the value of thermopower $S_{1}$ and the metal of which the float conductor is made has the value of thermopower $S_{2}$, respectively. When contacting, they form a cold junction of a thermocouple, whereas another junction is formed by a new conductor made from the same material as the float conductor and placed outside the container. This new conductor is supplied with a heating source. Since a current can be generated only in a closed circuit, in this case the physical conflict is solved in full: the current arises only on closing the contacts (SU A.c. 904 532).

If the solution can not be found after the final phase has been accomplished, AIPS recommends returning to the phase of the problem statement and conduct the problem analysis more carefully or select another subproblem. AIPS also includes special rules to help with managing this situation.

AIPS-85B includes nine sections. The full structure of AIPS-85B is depicted in Fig. 7.

\section{TIPS TECHNIQUES FROM A KNOWLEDGE- BASED POINT OF VIEW}

The main difficulty in automating the earliest design phases is that it has been unclear so far what knowledge to model in order to develop a knowledge base to support the automated reasoning process. As follows from studying inventive activities, ${ }^{10}$ to make inventions

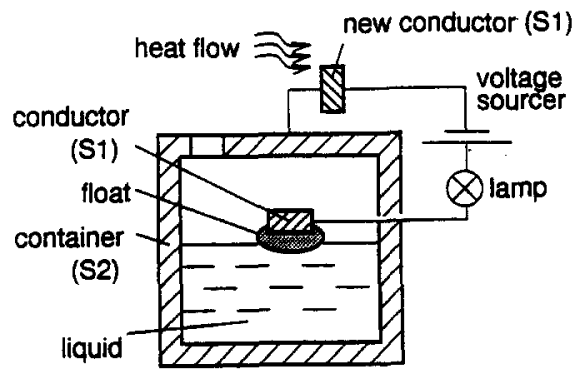

Fig. 6. Solution to the problem of preventing a spark jumping. 


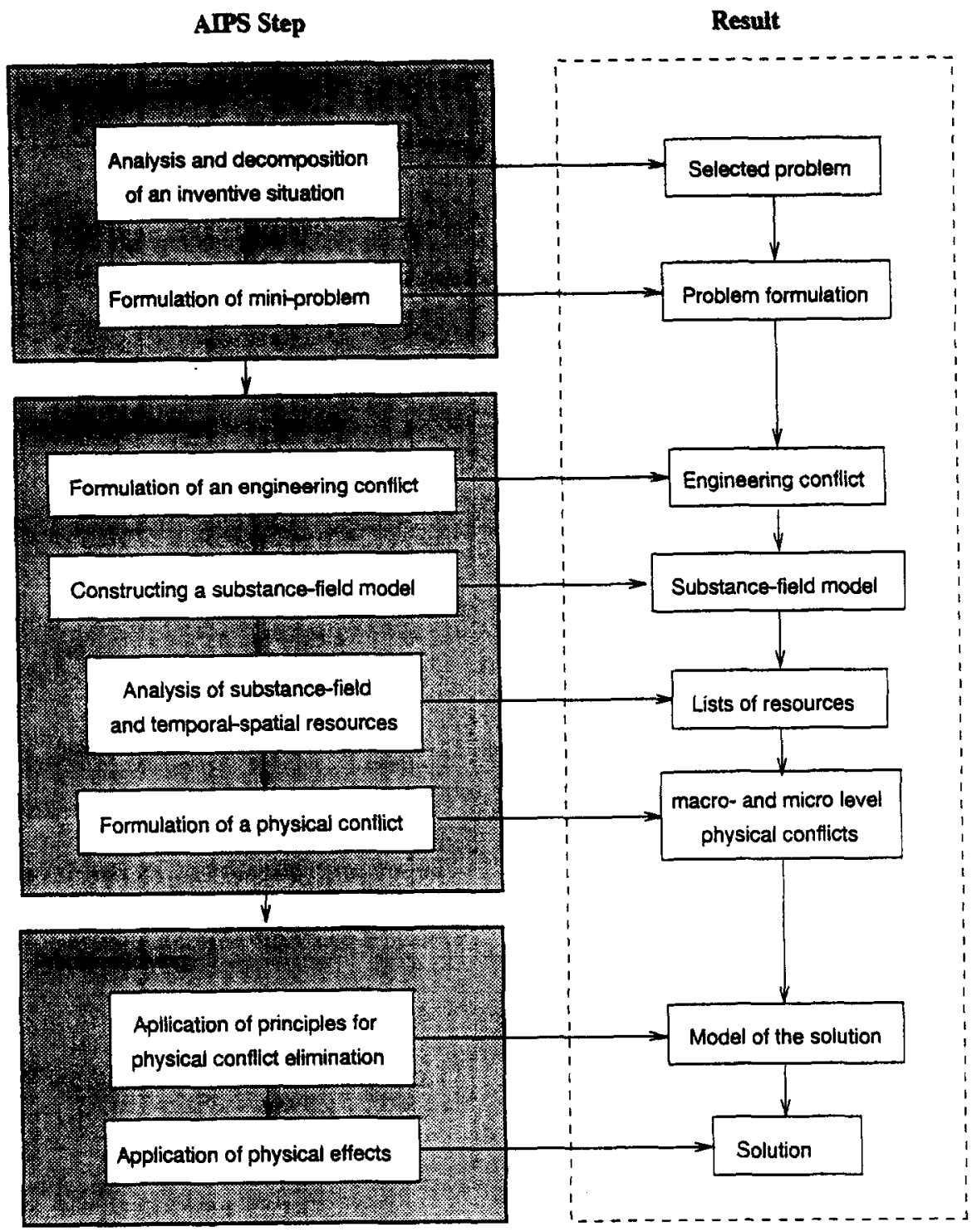

Fig. 7. Algorithm for inventive problem solving (AIPS).

the outstanding inventors use their own experience which is usually based on five to seven empirically found inventive principles and comprises knowledge from different engineering and scientific areas. Unfortunately, too much of this knowledge remains implicit.

To solve this problem by modeling all available design and scientific knowledge and developing a comprehensive knowledge base capable of storing all this knowledge is not possible because of the limitations in modern methods of knowledge representation and modeling techniques. In addition, even if the creation of such a knowledge base becomes possible, no formal method is known of manipulating this knowledge.

TIPS tackles this problem by studying high-level similarities in the inventive problem solving process between different domains. Many years of experience with using TIPS has shown that the similarities discovered can be successfully applied to solve virtually any inventive problem. For this reason, the knowledge collected in TIPS is considered to be one of the best knowledge sources for automating inventive design.

A general model of the problem solving process is depicted in Fig. 8. First, a problem is modeled either as a conflict between two engineering or physical parameters of a system or as a set of substance-field interactions within the system. Second, a suitable modification rule is applied to eliminate the conflict. As a result, an abstract model of the solution is constructed. The final step is to instantiate the model of the solution into a specific design to make the solution physically realizable and meeting initial functional requirements and satisfying all the constraints.

Since the universe of discourse in which TIPS operates is very large and covers almost every engineering domain, the search for a solution is organized at a high level of abstraction. This helps to significantly restrict the search space. However, this makes it very difficult to instantiate a solution model obtained into a 


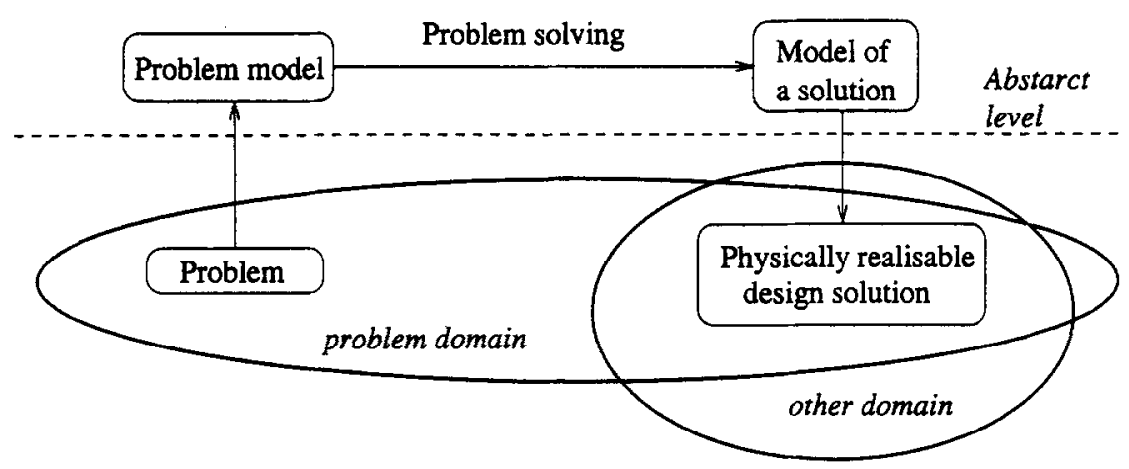

Fig. 8. Inventive problem solving in TIPS.

specific design. To tackle this problem, knowledge sources storing specific physical and design knowledge need to be developed. Besides, reasoning based on this knowledge can only be obtained by developing a technique for abstraction and by modeling different technical objects in a similar way.

\section{INVENTION MACHINE: COMPUTER SUPPORT OF TIPS}

The first attempts to automate TIPS were initiated in the Invention Machine Laboratory (IMLab, Minsk, Republic of Belarus) 5 years ago. ${ }^{18}$ Major efforts have been concentrated on developing a computer system to support an engineer in inventive activities by means of TIPS. The work resulted in the development of the Invention Machine ${ }^{\circledR}$ system, which is a well-indexed hypertext system containing three TIPS problem-solving techniques (inventive principles, inventive standards and collections of scientific-engineering effects) which are used independently. Each of these programs includes:

- A knowledge base consisting of specific rules for inventive problem solving.

- Industrial examples taken from patent collections. The examples illustrate how the same inventive principle can be applied in different engineering domains. It makes it easier to understand the principle and apply it to a new situation by analogy.

The invention machine has already been tested thoroughly on hundreds of problems in different engineering domains and has proven its effectiveness in many industrial companies and research institutes worldwide. The programs are used for educational purposes and provide assistance in inventive activities by giving advice on how to solve different engineering problems requiring an inventive approach. However, the programs only support TIPS and no real automated reasoning is yet performed. At the moment, the knowledge in the Invention Machine ${ }^{\circledR}$ is rather unstructured and consists of several overlapping parts. The knowledge needs to be restructured and integrated to enable automated reasoning.

\section{YMIR: A FRAMEWORK TO MODEL TIPS KNOWLEDGE}

Research focused on overcoming difficulties in modeling TIPS knowledge is currently conducted in the Knowledge-Based Systems Group of the University of Twente (Enschede, the Netherlands) in cooperation with the Invention Machine Laboratory. The goal of the joint project is to investigate how knowledge-based systems technology can be used to improve the Invention Machine ${ }^{\mathrm{A}}$. 19

To achieve this goal, YMIR ${ }^{3,20}$ is used, an ontology for engineering design, to model TIPS knowledge. The ontology defines an appropriate taxonomy of concepts for the formal description of design knowledge in different domains. The concepts in YMIR for the elements from which to synthesize technical system descriptions are called generic system models. These generalized concepts have been defined in terms of network models in system theory. ${ }^{21}$ Generic system models explicitly incorporate the relation between such features of an engineering system as behaviour and form. Multiple levels of abstraction with corresponding sets of generic system models are distinguished. The result allows for the gradual refinement of the design description at levels ranging from the original problem specification to the final artifact description.

YMIR contains a separate set of concepts for the structuring of synthesis knowledge. These concepts have been defined in terms of generic system models. A number of subtypes are distinguished which allow for a recursive description of the synthesis knowledge. The resulting collection of knowledge structure concepts makes it possible to systematically organize the knowledge to support its reuse.

The framework is applicable to all domains in which technical systems can be described as system-theoretical network models. Due to the explicit representation of the intrinsic relation between behaviour and form, the 
consequences of changes to the behaviour of a technical system for its form, or vice versa, can be determined instantly. Thus, a design description as defined by YMIR acts as an integrating model for the results of individual design actions in traditional CAD approaches.

In the authors' current research, they examine the applicability of YMIR concepts to modeling TIPS collections of physical effects. ${ }^{22}$

\section{CONCLUDING REMARKS AND FURTHER RESEARCH}

As shown above, TIPS includes several powerful techniques for inventive problem solving. These techniques are very strongly oriented to personal use; they suggest informal methods for problem solving with the use of heuristic rules for analysing inventive situations and searching for solutions. The final steps of the problem solving process - translating an abstract principle of a solution into a physically realizable design product - involve knowledge of different engineering domains as well as of natural sciences and currently is not supported by TIPS. As a consequence, the user of TIPS is supposed to possess a high degree of expertise in engineering design. The development of the Invention Machine ${ }^{(B)}$ by IMLab has shown that the use of methods for the development of conventional software makes it possible to create computer systems to support engineers in solving inventive problems. However, the role of the computer is a passive one: no machine reasoning takes place with the stored knowledge.

In the short run, the set of concepts, following TIPS, will be clarified to allow their use as the basis for a knowledge base on engineering design. This work has already started in connection with earlier work in the Knowledge-based Systems Group dedicated to the development of an ontology for engineering design. ${ }^{3}$ The long-term aim is to enable machine reasoning with the restructured and integrated knowledge base. They will try to establish a link with the framework for the later stages of engineering design already developed as a part of the STEVIN Project.

\section{REFERENCES}

1. Jones, J. C. Design Methods: Seeds of Human Futures. John Wiley, New York, 1981.

2. Suh, N. P. The Principles of Design. Oxford University Press, New York, 1990.
3. Alberts, L. K. YMIR: an ontology for engineering design. PhD thesis, University of Twente, Enschede, The Netherlands, 1993.

4. Welch, R. V. \& Dixon, J. R. Conceptual design of mechanical systems. In Proceedings of the 3rd International ASME Conference on Design Theory and Methodology, Phoenix, 1992, pp. 11-18.

5. Malmqvist, J. Computer-aided conceptual design of energy-transforming technical systems. In Proceedings of International Conference on Engineering Design ICED'93, The Hague, 1993, pp. 1541-50.

6. Tong, C. \& Sriram, D. (eds). Artificial Intelligence in Engineering Design, Vol. II: Models of Innovative Design, Reasoning about Physical Systems, and Reasoning about Geometry. Academic Press, New York, 1992.

7. Sycara, K. \& Navinchandra, D. Index transformation techniques for facilitating creative use of multiply cases. In Proceedings of the 12th International Joint Conference on Artificial Intelligence, Sydney, 1991.

8. Schmekel, H., A system for conceptual design based on general and systematic principles of design. Doctoral thesis, The Royal Institute of Technology, Stockholm, 1992.

9. Pahl, G. \& Beitz, W. Engineering Design: A Systematic Approach. Springer Verlag, Berlin, 1984.

10. Altshuller, G. S. \& Shapiro, R. V. About a technology of creativity. Questions of Psychology, 6 (1956) 37-49.

11. Altshuller, G. S. Creativity as an Exact Science. Gordon and Breach, New York, 1988.

12. Miles, L. D. Techniques of Value and Engineering. McGraw-Hill, New York, 1972.

13. Litvin, S. S. \& Guerassimov, V. M. Basic statements of conducting value-engineering analysis: recommendations. Journal of TRIZ, 2 (1992) 7-46 (in Russian).

14. Altshuller, G. S. To Find an Idea. Nauka, Novosibirsk, 1986 (in Russian).

15. Salamatov, Yu. P. Theory of engineering systems development. In Chance to Adventure, ed. A. Selutskii. Petrozavodsk, Karelia, 1991, pp. 7-174 (in Russian).

16. Vikentiev, I. L. \& Efremov, V. I., The curve rescues. In Games Rules Without Rules, ed. A. Selutskii. Petrozavodsk, Karelia, 1989 (in Russian).

17. Altshuller, G. S., History of AIPS. Journal of TRIZ, 1 (1992) 38-41 (in Russian).

18. Tsourikov, V. M. Inventive machine: second generation. AI \& Society, 7 (1) (1989) 62-78.

19. Mars, N. J. I., Sushkov, V. V. \& Wognum, P. M. AI and creative engineering design. In Proceedings of $A A A 193$ Spring Symposium on $A I$ and Creativity, Stanford, CA, 1993.

20. Alberts, L. K. YMIR: a domain-independent ontology for the formal representation of engineering design knowledge. In Proceedings of the IFIP WG 5.2 Workshop Formal Design Methods for CAD, Tallinn, Estonia, 1993.

21. Shearer, J. L., Murphy, A. T \& Richardson, H. H. Introduction To System Dynamics, Addison-Wesley, Reading, MA, 1967.

22. Sushkov, V. V., Reuse of physical knowledge in creative engineering design. In ECAI'94 Workshop Notes on Models and Techniques for Reuse of Designs, Amsterdam, 1994. 